\title{
GLOBAL ATTRACTIVITY FOR A POPULATION MODEL WITH TIME DELAY
}

\author{
JOSEPH W.-H. SO AND J. S. YU
}

(Communicated by Hal L. Smith)

\begin{abstract}
In this paper we give a sufficient condition which guarantees the global attractivity of the zero solution of a population growth equation.
\end{abstract}

\section{INTRODUCTION}

The delay differential equation

$$
N^{\prime}(t)=\beta N(t)\left[1-\frac{N(t-q)}{K}\right], \quad q>0,
$$

was proposed by Hutchinson [1] as a model for the growth of a single species. Using the change of variables $x(t)=\frac{N(q t)}{K}-1$ and defining $r=\beta q$, the above "Hutchinson's equation" can be reduced to

$$
x^{\prime}(t)=-r x(t-1)[1+x(t)]
$$

(cf. Wright [4]).

More realistically, we may assume that the growth rate $r$ depends on time. Thus consider the delay differential equation

$$
y^{\prime}(t)=-r(t)[1+y(t)] y(t-1), \quad t \geq 0,
$$

where $r:[0, \infty) \rightarrow(0, \infty)$ is continuous. This equation has been studied by Sugie [3] where it was shown that the zero solution of (1.1) is uniformly stable provided there exists a constant $0<r_{0}<\frac{3}{2}$ such that $r(t) \leq r_{0}$ for all $t \geq 0$.

In this paper, we are interested in the global attractivity of the zero solution. Due to the biological interpretation of (1.1), we are only interested in solutions $y(t)$ of (1.1) such that $y(t) \geq-1$ for $-1 \leq t \leq 0$ and $y(0)>-1$. In that case,

$$
1+y(t)=(1+y(0)) e^{-\int_{0}^{t} r(s) y(s-1) d s}>0
$$

and so $y(t)>-1$ for all $t \geq 0$.

We now state our main result.

Received by the editors October 1, 1993.

1991 Mathematics Subject Classification. Primary 34K20; Secondary 92D25.

Key words and phrases. Global attractivity, Hutchinson's equation.

This research was partially supported by the Natural Sciences and Engineering Research Council of Canada, grant number NSERC OGP36475. Research was done when the second author was visiting the University of Alberta.

(c) 1995 American Mathematical Society 
Theorem 1.1. If

$$
\int_{t-1}^{t} r(s) d s \leq \frac{3}{2} \quad \text { for all large } t
$$

and

$$
\int_{0}^{\infty} r(s) d s=\infty
$$

then every solution of (1.1) tends to zero as $t \rightarrow \infty$.

In the case when $r(t)$ is a constant $r_{0}$ and $0<r_{0} \leq \frac{3}{2}$, Theorem 1.1 was proved in Wright [4] (see also Kuang [2, Theorem 2.1, p.120]). It was conjectured in [4] (see also [2, Open Problem 4.1, p.171]) that the result is still true for $0<r_{0} \leq \frac{\pi}{2}$. This question remains open. In [2], it was conjectured (Open Problem 4.2, p.171) that under the assumption $0<r(t)<\frac{3}{2}$, every solution of (1.1) tends to zero. Theorem 1.1 gives a positive answer to this conjecture, provided (1.3) holds.

\section{Preliminaries}

Lemma 2.1. Let $0<\alpha<\frac{1}{2}$. The system of inequalities

$$
\left\{\begin{array}{l}
u \leq e^{v-\alpha v^{2}}-1 \\
v \leq 1-e^{-u-\alpha u^{2}}
\end{array}\right.
$$

has a unique solution: $(v, u)=(0,0)$ in the nonnegative quadrant $\{(v, u)$ : $v \geq 0, u \geq 0\}$.

Proof. Assume that (2.1) has another solution in the first quadrant of the $v-u$ plane besides $(0,0)$, say $\left(v_{0}, u_{0}\right)$. Then $u_{0}>0$ and $0<v_{0}<1$. Define $\Gamma_{1}$ to be the curve: $u=e^{v-\alpha v^{2}}-1$ and $\Gamma_{2}$ to be the curve: $v=1-e^{-u-\alpha u^{2}}$. Clearly $\left.\frac{d u}{d v}\right|_{(0,0)}=1,\left.\frac{d^{2} u}{d v^{2}}\right|_{(0,0)}=1-2 \alpha$ and $\left.\frac{d^{3} u}{d v^{3}}\right|_{(0,0)}=1-6 \alpha$ for $\Gamma_{1}$. On the other hand, $\left.\frac{d u}{d v}\right|_{(0,0)}=1,\left.\frac{d^{2} u}{d v^{2}}\right|_{(0,0)}=1-2 \alpha$ and $\frac{d^{3} u}{d v^{3}}=12 \alpha^{2}-6 \alpha+2$ for $\Gamma_{2}$. Hence $\Gamma_{2}$ lies above $\Gamma_{1}$ near $(0,0)$. The existence of $\left(v_{0}, u_{0}\right)$ implies that the curves $\Gamma_{1}$ and $\Gamma_{2}$ must intersect at a point in the first quadrant besides $(0,0)$. Let $\left(v_{1}, u_{1}\right)$ be the first such point, i.e. $v_{1}$ is smallest. Then the slope of $\Gamma_{1}$ at $\left(u_{1}, v_{1}\right)$ is no less than the slope of $\Gamma_{2}$ at $\left(u_{1}, v_{1}\right)$, i.e.

$$
\left(1-2 \alpha v_{1}\right) e^{v_{1}-\alpha v_{1}^{2}} \geq \frac{1}{1+2 \alpha u_{1}} e^{u_{1}+\alpha u_{1}^{2}}
$$

or

$$
\left(1-2 \alpha v_{1}\right)\left(1+2 \alpha u_{1}\right) \geq e^{u_{1}-v_{1}+\alpha\left(u_{1}^{2}+v_{1}^{2}\right)} .
$$

Claim: $u_{1}>v_{1}$.

Proof. Let $\phi(x)=1-e^{-x-\alpha x^{2}}-x$. Then $\phi(0)=0$ and $\phi^{\prime}(x)<0$ for $x>0$, since $2 \alpha<1$. Thus $\phi(x)<0$ for $x>0$ and $v_{1}=\phi\left(u_{1}\right)+u_{1}<u_{1}$.

Using the inequality $e^{x}>1+x \quad(x>0)$, we have

$$
1+2 \alpha\left(u_{1}-v_{1}\right)-4 \alpha^{2} u_{1} v_{1}>1+u_{1}-v_{1}+\alpha\left(u_{1}^{2}+v_{1}^{2}\right)
$$

or

$$
(-1+2 \alpha)\left(u_{1}-v_{1}\right)-4 \alpha^{2} u_{1} v_{1}>\alpha\left(u_{1}^{2}+v_{1}^{2}\right)
$$

which is a contradiction, since $0<\alpha<\frac{1}{2}$. This completes the proof. 
Lemma 2.2. If (1.3) holds, then every nonoscillatory solution of (1.1) tends to zero as $t \rightarrow \infty$.

Proof. Let $y(t)$ be an nonoscillatory solution of (1.1). Then there exists $t_{0}$ such that $y(t)$ is of one sign for $t \geq t_{0}$. Consider first the case $y(t) \leq 0$ for $t \geq t_{0}$. Since $1+y(t) \geq 0$, by $(1.1) y^{\prime}(t) \geq 0$. Thus $y(t)$ is increasing and $\lim _{t \rightarrow \infty} y(t)=-c$ exists. Clearly $-1<y\left(t_{0}\right) \leq-c \leq 0$. Integrating (1.1), we get (for $t>t_{0}+1$ )

$$
-\ln (1+y(t))+\ln \left(1+y\left(t_{0}+1\right)\right)=\int_{t_{0}+1}^{t} r(s) y(s-1) d s \leq-c \int_{t_{0}+1}^{t} r(s) d s .
$$

By (1.3), the right-hand side tends to $-\infty$ as $t \rightarrow \infty$ unless $c=0$. On the other hand, the left-hand side has a finite limit; therefore $c=0$. Hence $\lim _{t \rightarrow \infty} y(t)=0$. The case when $y(t)$ is eventually nonnegative is similar. This completes the proof.

The following lemma was essentially proved in [2, Theorem 3.1, p.128].

Lemma 2.3. Assume (1.2) holds. Let $y(t)$ be an oscillatory solution of (1.1). Then $y(t)$ is bounded above and is bounded below from -1 for $t \geq 0$.

Proof. Let $t_{0}>0$ be large enough so that (1.2) holds for all $t \geq t_{0}$. Let $t^{*}$ be a local maximum point of $y(t) \quad\left(t \geq t_{0}+1\right)$. Then $y^{\prime}\left(t^{*}\right)=0$ and by $(1.1)$ $y\left(t^{*}-1\right)=0$. Integrating $(1.1)$ from $t^{*}-1$ to $t^{*}$, we have

$$
1+y\left(t^{*}\right)=e^{-\int_{t^{*}-1}^{t^{*}} r(s) y(s-1) d s} .
$$

Since $y(s-1) \geq-1$, by $(1.2)$

$$
1+y\left(t^{*}\right) \leq e^{\int_{t^{*}-1}^{t^{*}} r(s) d s} \leq e^{\frac{3}{2}}
$$

and $y\left(t^{*}\right) \leq e^{\frac{3}{2}}-1$. Consequently, $\lim \sup _{t \rightarrow \infty} y(t) \leq e^{\frac{3}{2}}-1$.

Next, let $t_{*}$ be a local minimum point of $y(t)\left(t \geq t_{0}+3\right)$. Then $y^{\prime}\left(t_{*}\right)=0$ and $y\left(t_{*}-1\right)=0$. Proceeding similarly as before and using the fact that $y(s-1) \leq e^{\frac{3}{2}}-1$, we have

$$
1+y\left(t_{*}\right) \geq e^{\int_{t_{*}-1}^{t_{*}} r(s)\left(1-e^{\frac{3}{2}}\right) d s}=e^{-\left(e^{\frac{3}{2}}-1\right) \int_{t_{*}-1}^{t_{*}} r(s) d s} \geq e^{-\left(e^{\frac{3}{2}}-1\right) \frac{3}{2}} .
$$

Hence,

$$
y\left(t_{*}\right) \geq e^{-\frac{3\left(e^{\frac{3}{2}}-1\right)}{2}}-1,
$$

and $\liminf _{t \rightarrow \infty} y(t) \geq e^{-\frac{3}{2}\left(e^{\frac{3}{2}}-1\right)}-1>-1$.

\section{Proof of Theorem 1.1}

To complete the proof of Theorem 1.1, all we need is to show

Lemma 3.1. If (1.3) holds, then every oscillatory solution of (1.1) tends to zero as $t \rightarrow \infty$. 
Proof. Let $y(t)$ be an oscillatory solution of (1.1). By Lemma $2.3 y(t) \quad(t \geq 0)$ is bounded above and bounded below away from -1 . Let

$$
u=\limsup _{t \rightarrow \infty} y(t), \quad-v=\liminf _{t \rightarrow \infty} y(t) .
$$

Then $0 \leq v<1$ and $0 \leq u<\infty$. It suffices to show that $u=v=0$. For any $\epsilon$, choose $t_{0}=t_{0}(\epsilon)$ such that

$$
-v_{1} \equiv-v-\epsilon<y(t-1)<u+\epsilon \equiv u_{1}, \quad \text { for } t \geq t_{0} .
$$

We assume that $\epsilon$ is small enough so that $0<v_{1}<1$ and that $t_{0}$ is large enough so that (1.2) holds for $t \geq t_{0}-2$. Using (1.1), we have

$$
y^{\prime}(t) \leq r(t)[1+y(t)] v_{1}, \quad t \geq t_{0},
$$

and

$$
y^{\prime}(t) \geq-r(t)[1+y(t)] u_{1}, \quad t \geq t_{0}
$$

Let $\left\{t_{n}^{*}\right\}$ be an increasing sequence such that $t_{n}^{*} \geq t_{0}+1, y^{\prime}\left(t_{n}^{*}\right)=0, \lim _{n \rightarrow \infty} t_{n}^{*}$ $=\infty$ and $\lim _{n \rightarrow \infty} y\left(t_{n}^{*}\right)=u$. By $(1.1), y\left(t_{n}^{*}-1\right)=0$. For $t \in\left[t_{n}^{*}-1, t_{n}^{*}\right]$, we can integrate (3.3) from $t-1$ to $t_{n}^{*}-1$ and get

$$
-\ln [1+y(t-1)] \leq v_{1} \int_{t-1}^{t_{n}^{*}-1} r(s) d s
$$

or

$$
y(t-1) \geq-1+e^{-v_{1} \int_{t-1}^{t_{n}^{*}-1} r(s) d s} \quad \text { for } t \in\left[t_{n}^{*}-1, t_{n}^{*}\right] .
$$

By (1.1) it follows that

$$
y^{\prime}(t) \leq r(t)[1+y(t)]\left[1-e^{-v_{1} \int_{t-1}^{t_{n}^{*}-1} r(s) d s}\right], \quad t \in\left[t_{n}^{*}-1, t_{n}^{*}\right] .
$$

Combining this with (3.3), we have

$$
(\ln [1+y(t)])^{\prime} \leq \min \left\{r(t) v_{1}, r(t)\left[1-e^{-v_{1} \int_{t-1}^{t_{n}^{*}-1} r(s) d s}\right]\right\}, \quad t \in\left[t_{n}^{*}-1, t_{n}^{*}\right] .
$$

We will prove that

$$
\ln \left[1+y\left(t_{n}^{*}\right)\right] \leq v_{1}-\frac{1}{6} v_{1}^{2}
$$

There are two possibilities. 
Case 1: $\int_{t_{n}^{*}-1}^{t_{n}^{*}} r(s) d s \leq-\frac{\ln \left(1-v_{1}\right)}{v_{1}}$. Then by $(3.5)$

$$
\begin{aligned}
& \ln \left[1+y\left(t_{n}^{*}\right)\right] \\
& \leq \int_{t_{n}^{*}-1}^{t_{n}^{*}} r(t)\left[1-e^{-v_{1} \int_{t-1}^{t_{n}^{*}-1} r(s) d s}\right] d t \\
& =\int_{t_{n}^{*}-1}^{t_{n}^{*}} r(t)\left[1-e^{-v_{1} \int_{t-1}^{t} r(s) d s+v_{1} \int_{t_{n}^{*}-1}^{t} r(s) d s}\right] d t \\
& \leq \int_{t_{n}^{*}-1}^{t_{n}^{*}} r(t)\left[1-e^{-\frac{3}{2} v_{1}} e^{v_{1} \int_{t_{n}^{*}-1}^{t} r(s) d s}\right] d t \\
& =\int_{t_{n}^{*}-1}^{t_{n}^{*}} r(t) d t-e^{-\frac{3}{2} v_{1}} \int_{t_{n}^{*}-1}^{t_{n}^{*}} r(t) e^{v_{1} \int_{t_{n}^{*}-1}^{t} r(s) d s} d t \\
& =\int_{t_{n}^{*}-1}^{t_{n}^{*}} r(t) d t-e^{-\frac{3}{2} v_{1}} \frac{1}{v_{1}}\left[e^{v_{1} \int_{t_{n}^{*}-1}^{t_{*}^{*}} r(s) d s}-1\right] \\
& =\int_{t_{n}^{*}-1}^{t_{n}^{*}} r(t) d t-\frac{1}{v_{1}} e^{-v_{1}\left(\frac{3}{2}-\int_{t_{n}^{*}-1}^{t_{n}^{*}} r(s) d s\right)}\left[1-e^{-v_{1} \int_{i_{n}^{*}-1}^{t_{n}^{*}} r(s) d s}\right] .
\end{aligned}
$$

The function $\phi(x)=x-\frac{1}{v_{1}} e^{-v_{1}\left(\frac{3}{2}-x\right)}\left(1-e^{-v_{1} x}\right)$ is increasing for $0 \leq x \leq \frac{3}{2}$. Thus for $\int_{t_{n}^{*}-1}^{t_{n}^{*}} r(t) d t \leq-\frac{\ln \left(1-v_{1}\right)}{v_{1}} \leq \frac{3}{2}$, we have

$$
\begin{aligned}
\ln \left[1+y\left(t_{n}^{*}\right)\right] & \leq-\frac{\ln \left(1-v_{1}\right)}{v_{1}}-\frac{1}{v_{1}} e^{-v_{1}\left(\frac{3}{2}+\frac{\ln \left(1-v_{1}\right)}{v_{1}}\right)}\left[1-e^{\ln \left(1-v_{1}\right)}\right] \\
& =-\frac{\ln \left(1-v_{1}\right)}{v_{1}}-e^{-v_{1}\left(\frac{3}{2}+\frac{\ln \left(1-v_{1}\right)}{v_{1}}\right)} .
\end{aligned}
$$

Using the fact $e^{-x}>1-x$ for $x>0$, we have

$$
\ln \left[1+y\left(t_{n}^{*}\right)\right] \leq-1+\frac{3}{2} v_{1}-\frac{\left(1-v_{1}\right) \ln \left(1-v_{1}\right)}{v_{1}}<v_{1}-\frac{1}{6} v_{1}^{2}
$$

according to (2.21) on p.123 of [2].

For $\int_{t_{n}^{*}-1}^{t_{n}^{*}} r(t) d t \leq \frac{3}{2}<-\frac{\ln \left(1-v_{1}\right)}{v_{1}}$, we have

$$
\ln \left[1+y\left(t_{n}^{*}\right)\right] \leq \int_{t_{n}^{*}-1}^{t_{n}^{*}} r(t) d t-\frac{1}{v_{1}}\left[e^{-\frac{3}{2} v_{1}} e^{v_{1} \int_{t_{n}^{*}-1}^{t_{n}^{*}} r(t) d t}-e^{-\frac{3}{2} v_{1}}\right] .
$$

The function $x \mapsto x-\frac{1}{v_{1}} e^{-\frac{3}{2} v_{1}} e^{v_{1} x}$ is increasing for $0 \leq x \leq \frac{3}{2}$. Thus,

$$
\ln \left[1+y\left(t_{n}^{*}\right)\right] \leq \frac{3}{2}-\frac{1}{v_{1}}\left[1-e^{-\frac{3}{2} v_{1}}\right] \leq v_{1}-\frac{1}{6} v_{1}^{2}
$$

according to (2.19) on p.123 of [2]. 
Case 2: $-\frac{\ln \left(1-v_{1}\right)}{v_{1}}<\int_{t_{n}^{*}-1}^{t_{n}^{*}} r(s) d s \leq \frac{3}{2}$. Choose $\tau \in(0,1)$ such that

$$
\int_{t_{n}^{*}-\tau}^{t_{n}^{*}} r(s) d s=-\frac{\ln \left(1-v_{1}\right)}{v_{1}} \text {. }
$$

Then by (3.5) and (1.2),

$$
\begin{aligned}
& \ln \left(1+y\left(t_{n}^{*}\right)\right) \\
& \leq \int_{t_{n}^{*}-1}^{t_{n}^{*}-\tau} r(s) v_{1} d s+\int_{t_{n}^{*}-\tau}^{t_{n}^{*}} r(t)\left[1-e^{-v_{1} \int_{t_{n}-1}^{t_{n}^{*}-1} r(s) d s}\right] d t \\
& \leq v_{1} \int_{t_{n}^{*}-1}^{t_{n}^{*}-\tau} r(s) d s+\int_{t_{n}^{*}-\tau}^{t_{n}^{*}} r(s) d s-e^{-\frac{3}{2} v_{1}} \int_{t_{n}^{*}-\tau}^{t_{n}^{*}} r(t) e^{v_{1} \int_{t_{n}^{*}-1}^{t} r(s) d s} d t \\
& =v_{1} \int_{t_{n}^{*}-1}^{t_{n}^{*}-\tau} r(s) d s+\int_{t_{n}^{*}-\tau}^{t_{n}^{*}} r(s) d s-\frac{1}{v_{1}} e^{-\frac{3}{2} v_{1}}\left[e^{v_{1} \int_{t_{n}^{*}-1}^{t_{n}^{*}} r(s) d s}-e^{v_{1} \int_{t_{n}^{*}-1}^{t_{*}^{*}-\tau} r(s) d s}\right] \\
& =v_{1} \int_{t_{n}^{*}-1}^{t_{n}^{*}-\tau} r(s) d s+\int_{t_{n}^{*}-\tau}^{t_{n}^{*}} r(s) d s-\frac{1}{v_{1}} e^{-v_{1}\left(\frac{3}{2}-\int_{t_{n}^{*}-1}^{t_{n}^{*}} r(s) d s\right)}\left[1-e^{-v_{1} \int_{t_{n}^{*}-\tau}^{t_{n}^{*}} r(s) d s}\right] \\
& =v_{1} \int_{t_{n}^{*}-1}^{t_{n}^{*}-\tau} r(s) d s+\int_{t_{n}^{*}-\tau}^{t_{n}^{*}} r(s) d s-e^{-v_{1}\left(\frac{3}{2}-\int_{t_{n}^{*}-1}^{t_{n}^{*}} r(s) d s\right)} \\
& =v_{1} \int_{t_{n}^{*}-1}^{t_{n}^{*}} r(s) d s+\left(1-v_{1}\right) \int_{t_{n}^{*}-\tau}^{t_{n}^{*}} r(s) d s-e^{-v_{1}\left(\frac{3}{2}-\int_{t_{n}^{*}-1}^{t_{n}^{*}} r(s) d s\right)} \\
& \leq \frac{3}{2} v_{1}-\frac{\left(1-v_{1}\right) \ln \left(1-v_{1}\right)}{v_{1}}-1
\end{aligned}
$$

since $x \mapsto v_{1} x-e^{-v_{1}\left(\frac{3}{2}-x\right)}$ is increasing for $0 \leq x \leq \frac{3}{2}$. Thus, according to (2.21) on p.123 of [2],

$$
\ln \left(1+y\left(t_{n}^{*}\right)\right) \leq v_{1}-\frac{1}{6} v_{1}^{2} .
$$

Letting $n \rightarrow \infty$ and $\epsilon \rightarrow 0$, we have

$$
\ln (1+u) \leq v-\frac{1}{6} v^{2}
$$

Next, let $\left\{s_{n}^{*}\right\}$ be an increasing sequence such that $s_{n}^{*} \geq t_{0}+1, y^{\prime}\left(s_{n}^{*}\right)=0$, $\lim _{n \rightarrow \infty} y\left(s_{n}^{*}\right)=-v$ and $\lim _{n \rightarrow \infty} s_{n}^{*}=\infty$ By (1.1), $y\left(s_{n}^{*}-1\right)=0$. We will show that

$$
-\ln \left(1+y\left(s_{n}^{*}\right)\right) \leq u_{1}+\frac{1}{6} u_{1}^{2}
$$

For $t \in\left[s_{n}^{*}-1, s_{n}^{*}\right]$, integrating (3.4) from $t-1$ to $s_{n}^{*}-1$, we have

$$
\ln (1+y(t-1)) \leq u_{1} \int_{t-1}^{s_{n}^{*}-1} r(s) d s
$$

or

By (1.1)

$$
y(t-1) \leq-1+e^{u_{1} \int_{t-1}^{s_{n}^{*}-1} r(s) d s} .
$$

$$
[\ln (1+y(t))]^{\prime} \geq-r(t)\left[e^{u_{1} \int_{t-1}^{s_{n}^{*}-1} r(s) d s}-1\right] \quad \text { for all } t \in\left[s_{n}^{*}-1, s_{n}^{*}\right]
$$


GLOBAL ATTRACTIVITY FOR A POPULATION MODEL WITH TIME DELAY

2693

Case 1: $\int_{s_{n}^{*}-1}^{s_{n}^{*}} r(s) d s \leq 1$. Integrating (3.4) from $s_{n}^{*}-1$ to $s_{n}^{*}$, we have

$$
-\ln \left(1+y\left(s_{n}^{*}\right)\right) \leq u_{1} \int_{s_{n}^{*}-1}^{s_{n}^{*}} r(s) d s \leq u_{1} \leq u_{1}+\frac{1}{6} u_{1}^{2} .
$$

Case 2: $1<\int_{s_{n}^{*}-1}^{s_{n}^{*}} r(s) d s \leq \frac{3}{2}-\frac{\ln \left(1+u_{1}\right)}{u_{1}}$. Clearly $u_{1}>2$ in this case. As in Case 1, we have

$$
-\ln \left(1+y\left(s_{n}^{*}\right)\right) \leq u_{1} \int_{s_{n}^{*}-1}^{s_{n}^{*}} r(s) d s \leq \frac{3}{2} u_{1}-\ln \left(1+u_{1}\right) .
$$

Claim: $\frac{3}{2} u_{1}-\ln \left(1+u_{1}\right) \leq u_{1}+\frac{1}{6} u_{1}^{2}$. Indeed, if $u_{1} \geq 3$, then this inequality holds trivially. And for $2<u_{1}<3$,

$$
\frac{1}{2} u_{1}<\frac{3}{2}<\frac{2}{3}+\ln 3<\frac{1}{6} u_{1}^{2}+\ln \left(1+u_{1}\right) .
$$

Case 3: $\frac{3}{2}-\frac{\ln \left(1+u_{1}\right)}{u_{1}}<\int_{s_{n}^{*}-1}^{s_{*}^{*}} r(s) d s \leq \frac{3}{2}$. Choose $\tau \in(0,1)$ such that $\int_{s_{n}^{*}-1}^{s_{n}^{*}-\tau} r(s) d s=\frac{3}{2}-\frac{\ln \left(1+u_{1}\right)}{u_{1}}$. Then by (3.4) and (3.7), we have

$$
-\left(\ln [1+y(t)]^{\prime}\right) \leq \min \left\{r(t) u_{1}, r(t)\left[e^{u_{1} \int_{t-1}^{s_{n}^{*}-1} r(s) d s}-1\right]\right\} .
$$

Consequently

$$
\begin{aligned}
& -\ln \left(1+y\left(s_{n}^{*}\right)\right) \\
& \leq \int_{s_{n}^{*}-1}^{s_{n}^{*}-\tau} r(t) u_{1} d t+\int_{s_{n}^{*}-\tau}^{s_{n}^{*}} r(t)\left[e^{u_{1} \int_{t-1}^{s_{n}^{*}-1} r(s) d s}-1\right] d t \\
& \leq u_{1}\left(\frac{3}{2}-\frac{\ln \left(1+u_{1}\right)}{u_{1}}\right)+e^{\frac{3}{2} u_{1}} \int_{s_{n}^{*}-\tau}^{s_{n}^{*}} r(t) e^{-u_{1} \int_{s_{n}^{*}-1}^{t} r(s) d s}-\int_{s_{n}^{*}-\tau}^{s_{n}^{*}} r(t) d t \\
& =u_{1}\left(\frac{3}{2}-\frac{\ln \left(1+u_{1}\right)}{u_{1}}\right) \\
& +\frac{1}{u_{1}} e^{\frac{3}{2} u_{1}}\left[e^{-u_{1} \int_{s_{n}^{*}-1}^{s_{n}^{*}-\tau} r(s) d s}-e^{-u_{1} \int_{s_{n}^{*}-1}^{s_{*}^{*}} r(s) d s}\right]-\int_{s_{n}^{*}-\tau}^{s_{n}^{*}} r(t) d t \\
& =u_{1}\left(\frac{3}{2}-\frac{\ln \left(1+u_{1}\right)}{u_{1}}\right) \\
& +\frac{1}{u_{1}}\left[e^{u_{1}\left(\frac{3}{2}-\int_{s_{n}^{*}-1}^{s_{*}^{*}-\tau} r(s) d s\right)}-e^{u_{1}\left(\frac{3}{2}-\int_{s_{n}^{*}-1}^{s_{*}^{*}} r(s) d s\right)}\right]-\int_{s_{n}^{*}-\tau}^{s_{n}^{*}} r(t) d t \\
& \leq u_{1}\left(\frac{3}{2}-\frac{\ln \left(1+u_{1}\right)}{u_{1}}\right) \\
& +\frac{1}{u_{1}}\left[1+u_{1}-1-u_{1}\left(\frac{3}{2}-\int_{s_{n}^{*}-1}^{s_{n}^{*}} r(s) d s\right)\right]-\int_{s_{n}^{*}-\tau}^{s_{n}^{*}} r(t) d t
\end{aligned}
$$


due to the choice of $\tau$ and because $e^{x} \geq 1+x$ for $x \geq 0$. Thus,

$$
\begin{aligned}
& -\ln \left(1+y\left(s_{n}^{*}\right)\right) \\
& \quad \leq u_{1}\left(\frac{3}{2}-\frac{\ln \left(1+u_{1}\right)}{u_{1}}\right)+1-\frac{3}{2}+\int_{s_{n}^{*}-1}^{s_{n}^{*}} r(s) d s-\int_{s_{n}^{*}-\tau}^{s_{n}^{*}} r(t) d t \\
& \quad=u_{1}\left(\frac{3}{2}-\frac{\ln \left(1+u_{1}\right)}{u_{1}}\right)-\frac{1}{2}+\int_{s_{n}^{*}-1}^{s_{n}^{*}-\tau} r(s) d s \\
& \quad \leq 1-\ln \left(1+u_{1}\right)+\frac{3}{2} u_{1}-\frac{\ln \left(1+u_{1}\right)}{u_{1}} \\
& \quad=1-\frac{\left(1+u_{1}\right) \ln \left(1+u_{1}\right)}{u_{1}}+\frac{3}{2} u_{1} \\
& \leq u_{1}+\frac{1}{6} u_{1}^{2}
\end{aligned}
$$

by (2.22) on p.123 of [2].

Thus we have shown that

$$
-\ln \left(1+y\left(s_{n}^{*}\right)\right) \leq u_{1}+\frac{1}{6} u_{1}^{2} .
$$

Letting $n \rightarrow \infty$ and $\epsilon \rightarrow 0$, we have

$$
-\ln (1-v) \leq u+\frac{1}{6} u^{2}
$$

or

$$
1-v \geq e^{-u-\frac{1}{6} u^{2}}
$$

Since $u, v$ satisfy the inequalities in (2.1) with $\alpha=\frac{1}{6}$, by Lemma 2.1 $u=v=0$. This completes the proof.

\section{ACKNOWLEDGMENT}

The authors would like to thank Professor Yang Kuang (Arizona State University) for a careful reading of a previous version of this paper. His comments helped strengthen our main result.

\section{REFERENCES}

1. G.E. Hutchinson, Circular causal systems in ecology, Ann. New York Acad. Sci. 50 (1948), 221-246.

2. Y. Kuang, Delay differential equations with applications in population dynamics, Academic Press, Boston, 1993.

3. J. Sugie, On the stability for a population growth equation with time delay, Proc. Roy. Soc. Edinburgh Sect. A 120 (1992), 179-184.

4. E.M. Wright, A non-linear difference-differential equation, J. Reine Angew. Math. 194 (1955), 66-87.

Department of Mathematical Sciences, University of Alberta, Edmonton, Alberta, CANADA T6G 2G1

E-mail address: joso@jazz.math.ualberta.ca

Department of Applied Mathematics, Hunan University, Changsha, Hunan 410082, PeOple's Republic of China 